\section{Caryocinèses aberrantes répétées conduisant à la stérilité mâle par l'action d'un facteur mendélien}

Epilobium spicatum Lmk. (= Chamaenerium angustifolium Scop.) présente une forme à fleurs blanches qu'on peut observer dans la nature; celle qui a fait l'objet de la présente étude, est apparue dans une population issue de graines d'origine inconnue.

\section{Description des phénotypes}

Le type normal, à fleurs pourpre-clair, se caractérise par la présence de pigment dans toutes les pièces florales ainsi que dans l'appareil végétatif.

Le type albiflora est totalement dépourvu de pigment pourpre: les fleurs sont blanches et l'ensemble de la plante d'un vert clair. Le pigment s'élabore très tôt dans la vie de la plante, il est déjà visible dans l'axe hypocotylaire des plantules et dans les feuilles de rosette.

Dans tous les individus de type albiflora, l'absence de pigment s'accompagne de stérilité pollinique alors que le type normal produit un pollen toujours régulier. La stérilité cependant n'affecte que le sexe mâle et la fertilité des ovules n'est pas abaissée.

\section{Analyse de la transmission}

D'après les premiers résultats, le déterminisme de ce double phénotype de stérilité mâle associée à l'albinisme se comporte comme monofactoriel (ségrégation $3 / 1$ après autofécondation, $1 / 1$ après croisement-test). I_es résultats ultérieurs montreront s'il s'agit de l'action pléiotropique d'un gène ou de 2 gènes non encore disjoints.

\section{Mécanisme inducteur de la stérilité pollinique}

Le développement du pollen a été étudié comparativement dans $l \approx$ type normal et le type albiflora. Les deux divisions de méiose se déroulent régulièrement dans les plantes à fleurs blanches comme dans les plantes normales. 18 bivalents montrent une disjonction réguliere en anaphase. En fin de méiose, chacun des 4 noyaux formés évolue vers un état quiescent.

Dans les plantes normales, le cloisonnement qui intervient à ce stade conduit à la formation de tétrades régulières. Dans le type albiflora, il n'y a pas cytocinèse à la fin de la méiose, mais une nouvelle division se prépare dans chacun des 4 noyaux. Tandis que le nucléole se résorbe, les chromosomes reprennent leur individualité et redeviennent trapus, presque ponctiformes. L'évolution chromatique semble normale au niveau des chromosomes, mais on ne peut observer ni fissuration, ni appariement. Après cette courte prophase, apparaît un fuseau sur lequel se distribuent les éléments chromatiques et sans qu'on puisse caractériser de métaphase, un mouvement anaphasique les répartit au hasard à chacun des 2 pôles (Fig. a). Le nombre total sur un fuseau est toujours de l'ordre du nombre haploïde 18: des chromosomes entiers sont donc redistribués passivement aux pôles. Cette division aberrante se termine par lc retour à l'état quiescent et, au sein de chaque groupe de chromosomes ainsi constitue, un nucléole apparaît; une cytocinèse isole ensuite une huitaine de cellules.

Elles sont le siège de nouvelles divisions, en tous points semblables à la précédente, mais où apparaissent des chromosomes en nombre variable, nettement inférieur à 18. Ils se distribuent au hasard, et une cytocinèse partage la cellule en 2, parfois 3 éléments, de taille souvent inégale, les plus petits ne renfermant plus qu'un seul chromosome. Le nombre de divisions successives dans ces microcytes ne peut être déterminé avec précision; chacune étant suivie de cytocinèse, il en résulte la formation de "tétrades" à éléments multiples, renfermant jusqu'à une vingtaine de microcytes de taille très inégale (Fig. b).

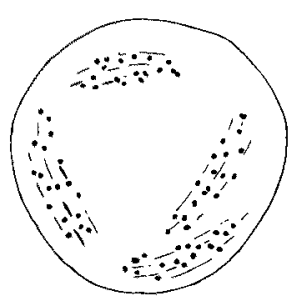

Fig. $a$

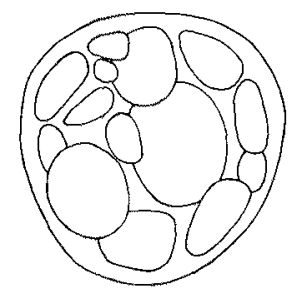

Iij. $b$
Ces derniers amorcent une évolution normale vers les grains de pollen, et élaborent même une membrane épaissie. Mais ils ne tardent pas à s'arrêter dans leur développement et à dégénérer. A la déhiscence des anthères, le pollen émis ne se réduit plus qu'à des enveloppes presque toutes vides.

Ces faits suggèrent quelques remarques: c'est en agissant sur des processus caryologiques qu'un facteur récessif détermine la stérilité mâle. Il se manifeste par une action double: induction de divisions aberrantes, et répétition du phénomène. Le déroulement de ces divisions constitue un fait très remarquable. Il montre que peuvent être dissociées l'évolution morphologique des chromosomes et la cinétique de caryocinèse : bien qu'entrainées et distribuées aux pôles, les unités chromosomiques n'ont pas eu d'évolution prophasique normale. Le fait caractéristique de ces divisions aberrantes est la distribution au hasard de chromosomes sans fissuration ni appariement. Déclanché déjà dans la cellule-mère, à la fin de la méiose, ce processus se répète dans les microcytes.

Le cas présent rappelle celui de po ("polymitotic"), gène défini et bien analysé dans le Maïs par BEADLE ${ }^{1}$. Si le mode d'action de "po" diffère par une stérilité non exclusive du sexe mâle et le fait qu'il agit, après une méiose achevée, sur des microspores constituées, les aspects cytologiques sont comparables dans leurs singularites: déclanchement de divisions nouvelles aberrantes, répétition du phénomène, répartition de chromosomes non divisés sur les fuseaux.

J. BRUN

Institut de Botanique de la Faculté des Sciences de Strasbourg (France), le 70 décembre 1957.

\section{Zusammenfassung}

Bei der weissblühenden Rasse von Epilobium spicatum Lmk. wurde Pollensterilität beobachtet. Der Sterilitätsfaktor ist rezessiv an die Albinoform gebunden und löst in den Pollenmutterzellen wiederholte, abweichende, postmeiotische Karyokinesen aus. . Diese beruhen auf einer Chromosomenverteilung ohne vorhergehende Paarung oder Verdoppelung.

1 G. W. BEADLE, A gene in maize for supernumerary cell divisions following meiosis (Cornell Univ. Agr. Exp. Sta. Memoir 135 [1931]) 